\title{
Endoscopic Endonasal Management of Recurrent Petrous Apex Cholesterol Granuloma
}

\author{
Nancy McLaughlin ${ }^{1}$ Daniel F. Kelly ${ }^{1} \quad$ Daniel M. Prevedello ${ }^{2}$ Kiarash Shahlaie $^{1} \quad$ Ricardo L. Carrau $^{3}$ \\ Amin B. Kassam ${ }^{4}$
}

${ }^{1}$ Brain Tumor Center, John Wayne Cancer Institute of Saint John's Health Center, Santa Monica, California, United States

2 Department of Neurological Surgery, The Ohio State University, Columbus, Ohio, United States

${ }^{3}$ Department of Head and Neck Surgery, The Ohio State University, Columbus, Ohio, United States

${ }^{4}$ Department of Surgery, Division of Neurosurgery, University of Ottawa, Ontario, Canada

J Neurol Surg B 2012;73:190-196.

\author{
Address for correspondence and reprint requests Amin B. Kassam, \\ M.D., F.R.C.S.C., Neurosurgery Division, The Ottawa Hospital - Civic \\ Campus, 1053 Carling Avenue, Ottawa, Canada K1Y 4E9 \\ (e-mail: kassamab@gmail.com).
}

\section{Introduction}

Cholesterol granulomas (CGs) are inflammatory lesions that occur rarely in the petrous apex. ${ }^{1}$ Initially, these lesions tend to remain asymptomatic. As they expand, headaches and cranial neuropathies may arise. ${ }^{1,2}$ The treatment of symptomatic petrous apex CG (PACG) is surgical drainage of the cyst and permanent aeration of petrous apex air cells to prevent recurrence. ${ }^{1,3}$ Several transcranial approaches have been used to treat these lesions, the choice of which depends on the patient's preoperative hearing status and the lesion's location in relation to critical neurovascular structures. ${ }^{2-5}$ These traditional lateral skull base approaches expose to potential structural damage to the inner ear and facial nerve as well as added morbidities of any intracranial procedure. ${ }^{4}$ They also have the shortcoming of often producing a relatively narrow bony opening, potentially compromising long-term drainage, and preventing the use of stents between the aerated cavity and the sphenoid sinus. In addition to having recurrence rates as high as $60 \%$, repeat transcranial surgeries for recurrent lesions may be associated with significant received

August 7, 2010

accepted

November 15, 2010

published online

May 17, 2012
Copyright $\odot 2012$ by Thieme Medical

Publishers, Inc., 333 Seventh Avenue, New York, NY 10001, USA.

Tel: +1(212) 584-4662.
DOI http://dx.doi.org/ $10.1055 / \mathrm{s}-0032-1312706$ ISSN 2193-6331. 


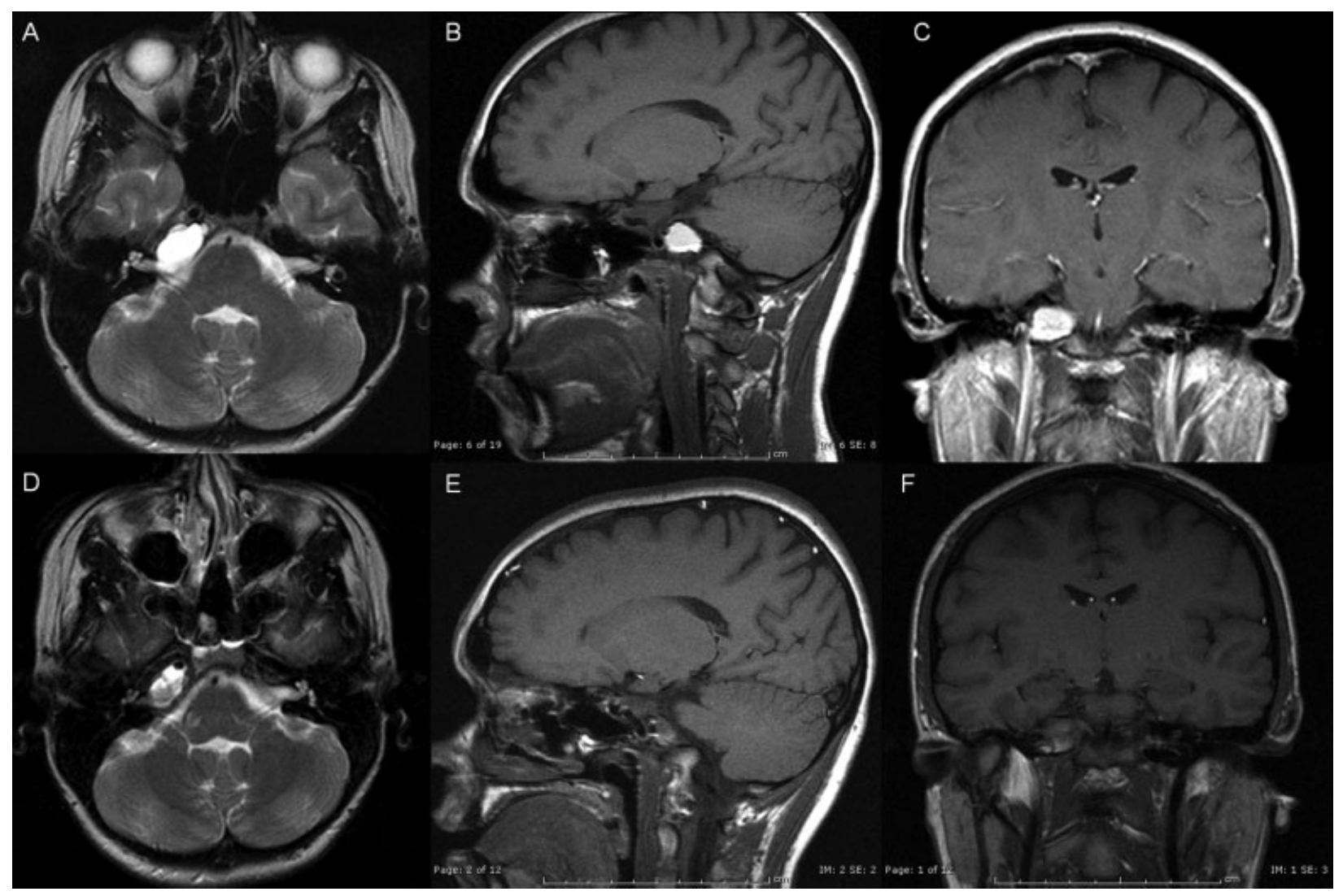

Figure 1 Preoperative (top row) and postoperative (bottom row) magnetic resonance imaging in T2-weighted image axial (A, D), T1-weighted image sagittal (B, E), and T1-weighted image postgadolinium coronal (C, F) views.

morbidities. ${ }^{2,3}$ Recently, the endoscopic endonasal approach has gained popularity in the neurosurgical community. This less invasive approach is attractive for the initial surgical management of symptomatic PACG and possibly even more so for symptomatic recurrent PACG.

In this study, we describe a case of recurrent symptomatic PACG treated by an expanded endonasal approach. We review all reported cases treated by an endoscopic endonasal approach and attempt to establish management pearls regarding the endoscopic endonasal management of PACG.

\section{Case Report and Technique Description}

A 19-year-old woman presented with an ongoing severe headache of subacute onset and 3-day history of diplopia. Physical examination was unremarkable except for right abducens nerve palsy. Head computed tomography scan demonstrated expansion of the right petrous apex. Magnetic resonance imaging showed a right petrous apex mass measuring $25 \times 14 \times 14 \mathrm{~mm}$ abutting the posterior wall of the sphenoid sinus. The lesion was hyperintense on both T1- and T2-weighted images and did not enhance following gadolinium administration (-Fig. 1). Given her rapid onset of symptoms, surgical drainage was recommended.

The patient underwent an endonasal transsphenoidal approach using intermittently the operating microscope and the endoscope for visualization. The location of the lesion was confirmed in the lower inferior right aspect of the sphenoid sinus with intraoperative neuronavigation. After creating a wide bony opening to the face of the CG, its capsule was incised. Characteristic greenish fluid with cholesterol crystals poured forth. After satisfactory drainage, the bony opening was widened and multiple fragments of the capsule were removed. Final inspection of the cyst's cavity showed complete drainage of the lesion. Postoperatively, symptoms progressively resolved.

Four months after initial surgery, clinicoradiological recurrence occurred and mandated a repeat transsphenoidal and infrapetrous approaches (-Fig. 2). After removing scar tissue from the prior bony opening in the sphenoid floor, care was taken to identify the vertical and horizontal segments of the internal carotid artery (ICA). The high speed drill was used to widen the bony opening inferior to the petrous carotid into the petrous apex in a medial and inferior direction. Once exposed on a large surface, the anterior wall of the cyst was removed, the content of the cyst was drained, and its lining partly removed. A 45-degree endoscope allowed visualization of the empty cavity (-Fig. 3). A Doyle splint was inserted into the cyst's cavity on $\sim 1 \mathrm{~cm}$ and extended out into the sphenoid sinus, maintaining patency during the healing process. At 3 months after surgery, the Doyle splint was removed under endoscopic visualization. A healed cylindrical communication between the involved petrous apex cells and the sphenoid sinus was appreciated ( - Fig. 4). A rubber 
192 Endoscopic Endonasal Management of Recurrent Petrous Apex CG McLaughlin et al.

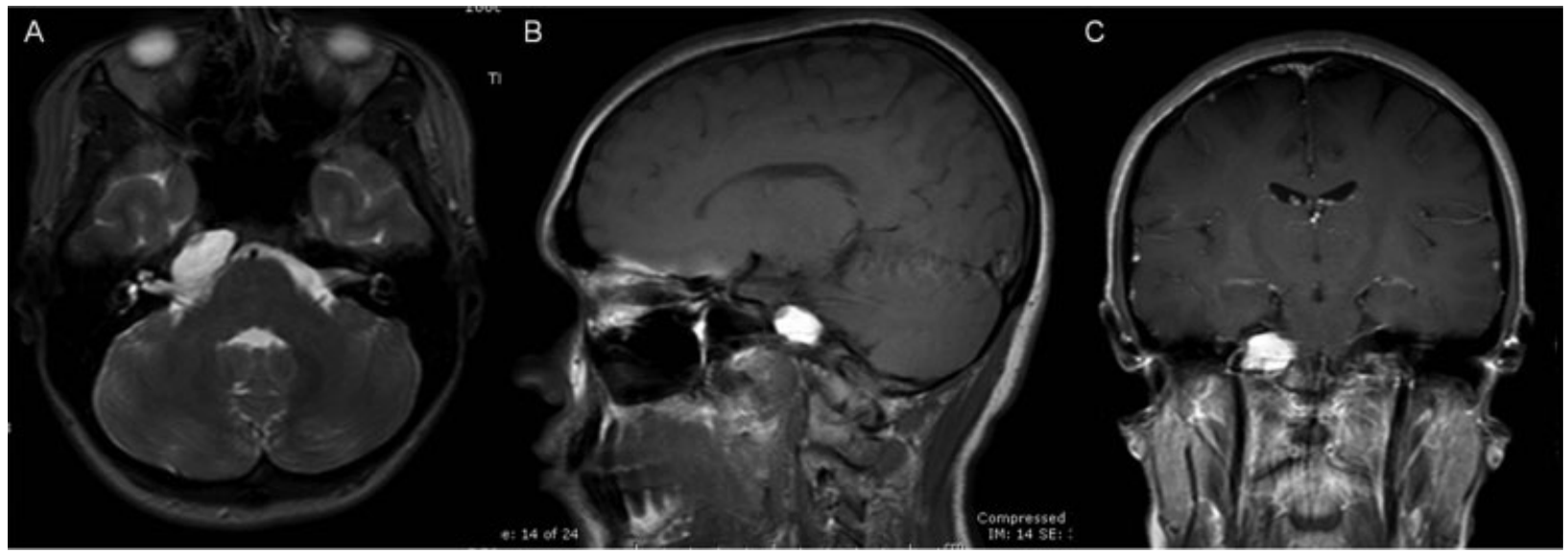

Figure 2 Magnetic resonance imaging at clinical recurrence in T2-weighted image axial (A), T1-weighted image sagittal (B), and T1-weighted image postgadolinium coronal (C) views.

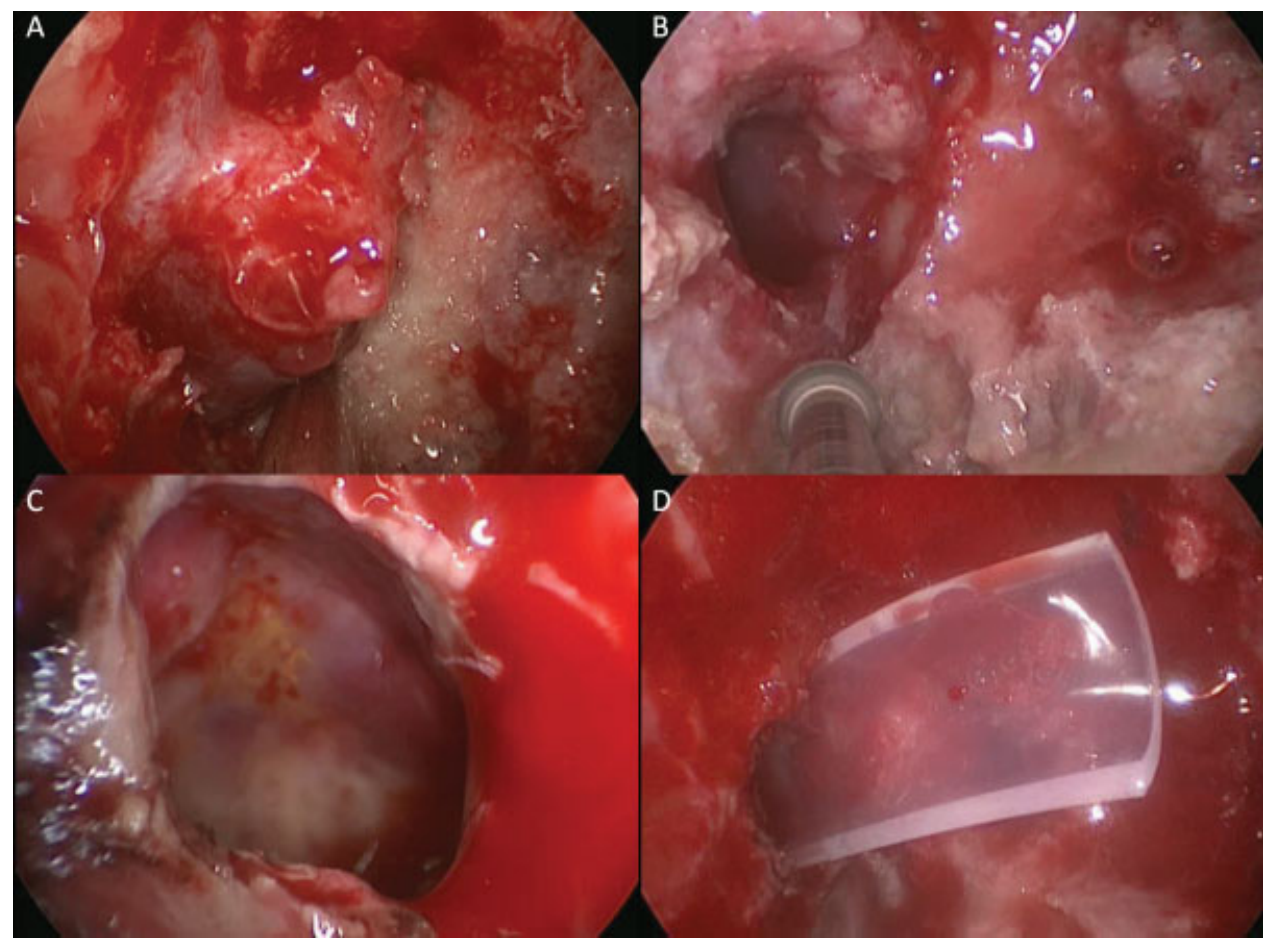

Figure 3 Intraoperative view of the repeat transsphenoidal and infrapetrous approaches. (A) Exposure of the petrous apex and cyst on a large surface. (B) Widening of the bony opening inferior to the petrous carotid into the petrous apex in a medial and inferior direction. (C) Visualization of the emptied cavity with a 45-degree endoscope. (D) Insertion of a Doyle splint into the cyst's cavity.

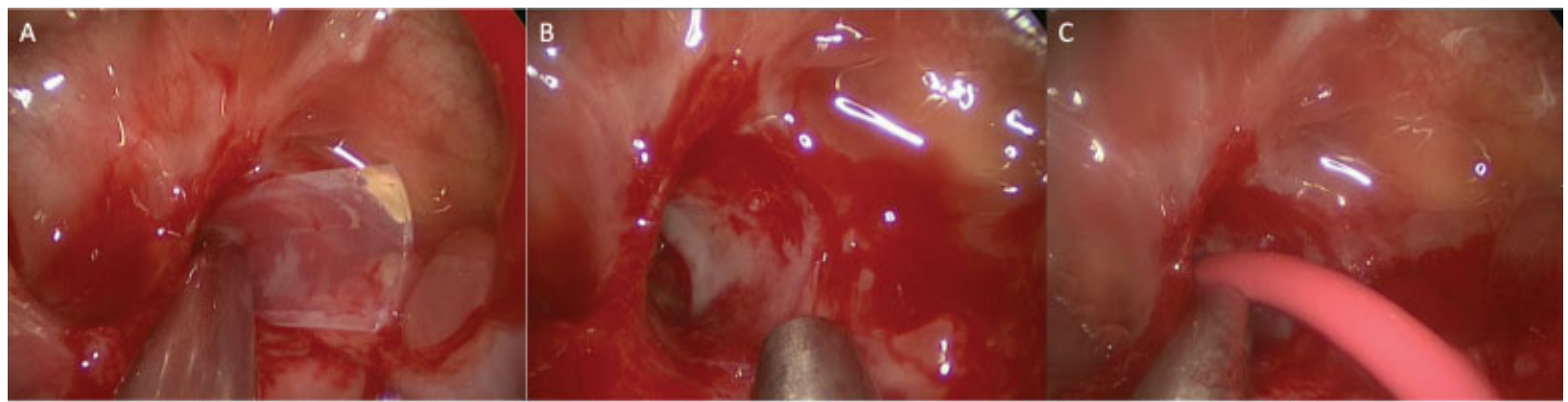

Figure 4 Intraoperative view of the removal of the Doyle splint with irrigation of the cavity. 


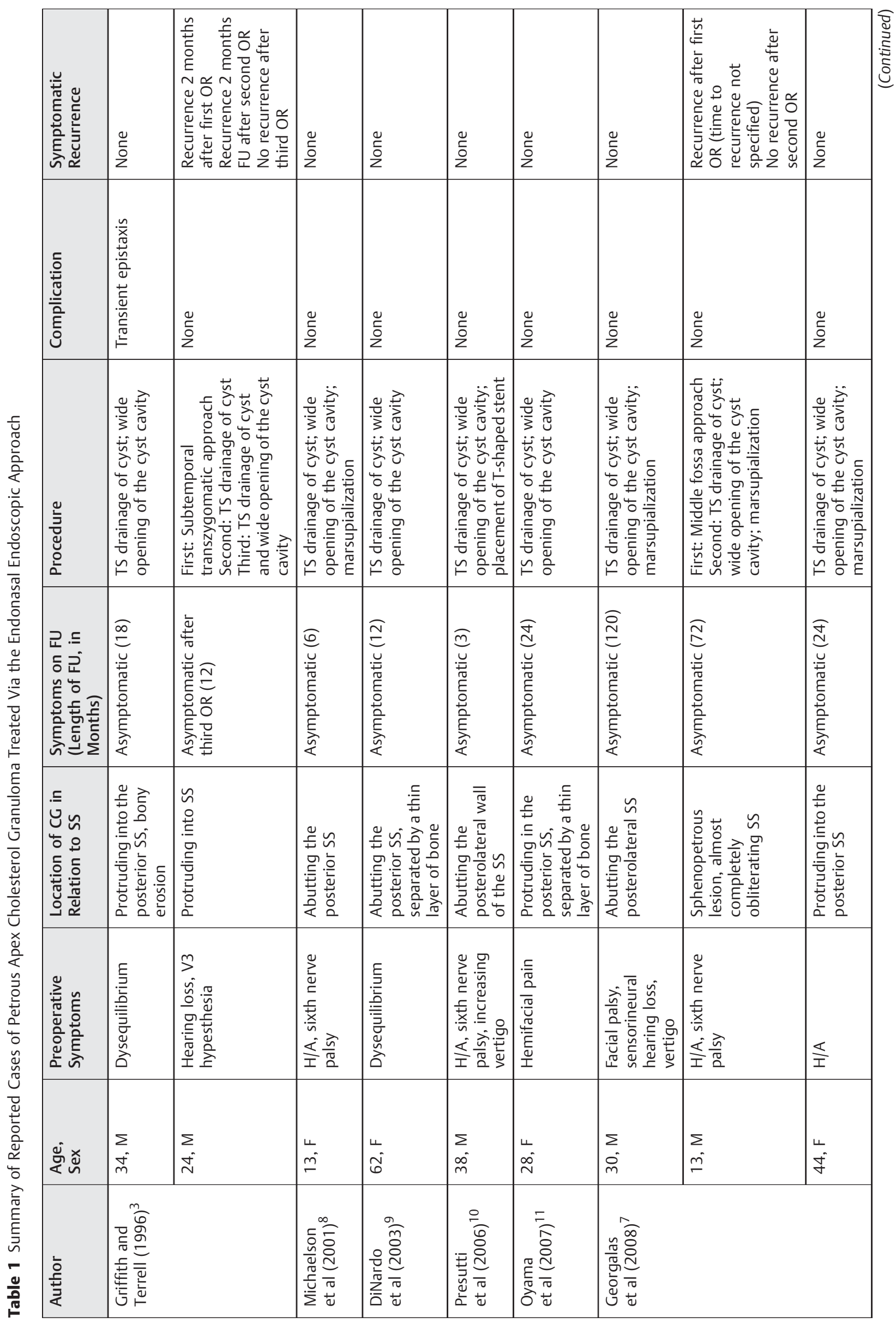




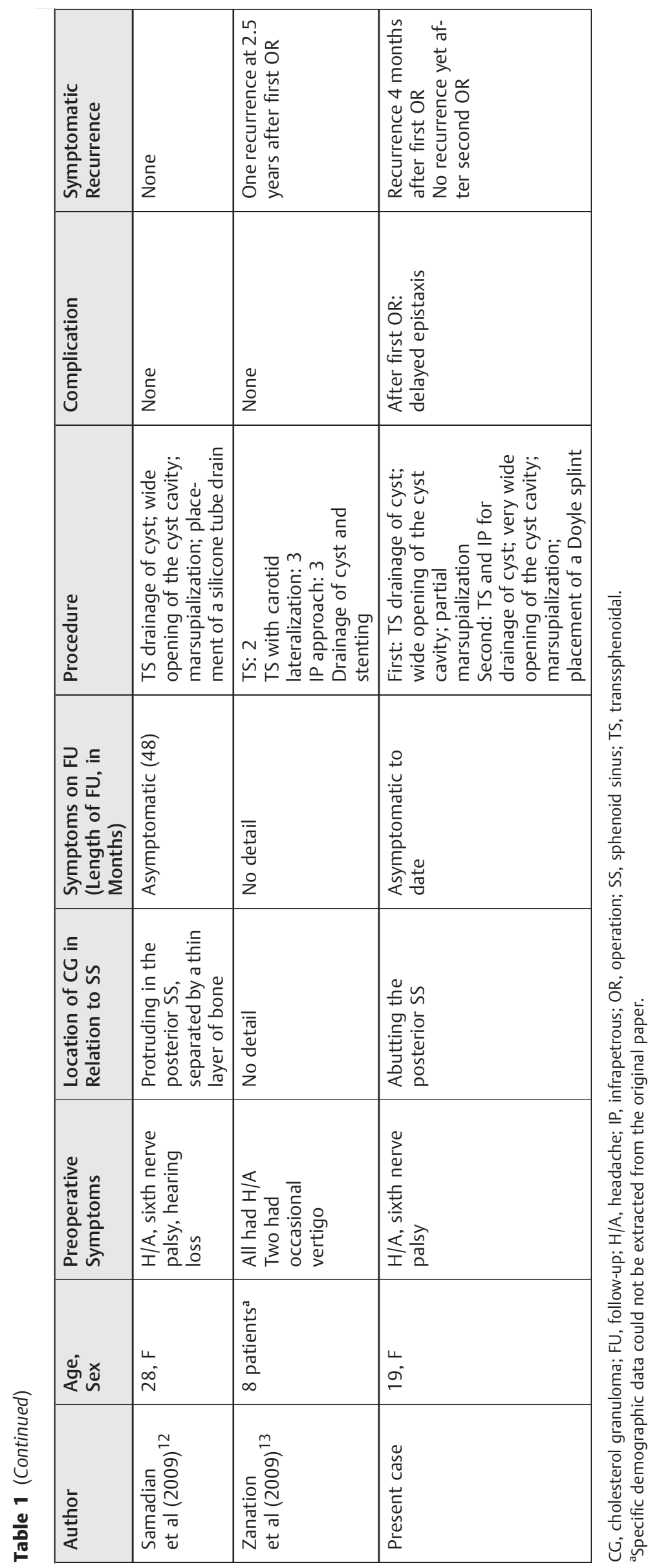


catheter was used to irrigate the aerated cavity. The patient currently remains symptom-free and recurrence-free.

\section{Discussion}

CGs rarely occur in the petrous apex because the petrous apex is pneumatized in only $30 \%$ of temporal bones. ${ }^{6}$ They result from obstruction of the normal aeration of petrous apex air cells. This obstruction is believed to lead to negative pressure within the air cells, eventually causing mucosal edema, tissue breakdown, and hemorrhage and with accumulation of breakdown products such as cholesterol. ${ }^{1,2}$ The cholesterol crystals induce a granulomatous inflammatory reaction resulting in the typical CG.

For symptomatic patients, surgery is presently the only treatment option available. Surgical treatment implies cyst drainage, with or without removal of its lining, and restoration of air cell aeration. Some authors have also proposed complete excision of the lesion followed by obliteration of the cavity with vascularized tissue. ${ }^{2}$ Numerous transcranial approaches have been performed to treat PACG such as middle cranial fossa approaches and lateral skull base approaches including the translabyrinthine, infratrochlear, infralabyrinthine, transcochlear approaches. ${ }^{2-5}$ The specific approach recommended depended on the patient's hearing status and the cyst's location. Given the lateral trajectory of transcranial skull base approaches, they inevitably expose to potential structural damage to the inner ear and facial nerve as well as added morbidities of any intracranial procedure., ${ }^{4,7}$

In addition, although satisfactory cyst drainage may be accomplished through these transcranial routes, bony opening obtained is often relatively narrow, rendering difficult intraoperative splinting techniques and potentially putting at risk chronic drainage. Indeed, recurrences have been reported to be as high as $60 \%$ irrespective of the used transcranial approach and degree of completeness of removal. ${ }^{2,3}$ Stenosis of the opening used to exteriorize the cyst may result in reaccumulation of its content and recurrence of symptoms. Just as for initial procedures, revision transcranial surgeries for recurrent lesions may also be associated with significant morbidities.

Contemporary progresses in endoscopic surgery as well as in image-guidance technology have allowed treatment of PACG through a less invasive route. Petrous lesions located medial to the ICA and that abut, protrude, or invade the sphenoid sinus may be safely approached through a transsphenoidal approach ${ }^{3,7-13}$ (-Table 1). The use of the endoscope as visualization technique has enabled a more complete drainage of the cyst. ${ }^{14}$ The cyst cavity may be closely inspected under endoscopic visualization to remove septations between separate fluid loculations or any remote debris. ${ }^{14}$

Including the present case, 19 cases of PACG have been treated through an endoscopic endonasal approach (-Table 1). $)^{3,7-13}$ Of the 19 patients, 16 have been drained initially through a medial transsphenoidal approach (with or without lateralization of the ICA). Of these 16 patients, 3 developed a symptomatic recurrence that required repeat surgery. The remaining three patients had been drained initially via an infrapetrous approach, and did not develop a recurrence. ${ }^{13}$ Accordingly, 3 of the 19 patients (15.7\%) with PACG treated via an endonasal route presented a recurrence at 2,4 , and 30 months postoperative. This recurrence rate (15.7\%) is significantly lower than that reported following other transcranial approaches (up to 60\%). Several factors may contribute to this lower recurrence rate including a wider bony opening and, consequently, a more extensive removal of the cyst's anterior or medial wall, afforded by the endonasal endoscopic approaches (especially with the infrapetrous approach). In addition, the superior visualization of the cavity using angled endoscopes enables to assess and address remaining septations and/or debris; therefore, optimizing the drainage. ${ }^{14}$ Furthermore, the use of a stent to preserve a drainage pathway throughout the healing process may contribute to reducing recurrences. This is especially important with the infrapetrous approach since the path of dissection is deeper than that performed in the medial transsphenoidal approach, and therefore may be more at risk of scarring. ${ }^{13}$ All of these considerations are of utmost importance when treating a recurrent PACG. We favor the endonasal endoscopic infrapetrous approach for recurrent PACG regardless of the initial surgical approach. In comparison to a transcranial surgery which may be associated with significant morbidity, no complications have yet occurred following endonasal endoscopic treatment for recurrent PACG.

\section{Conclusion}

Overall, the endoscopic endonasal route enables treatment of PACG through an anterior trajectory, preserving hearing, vestibular, and facial function. This approach is significantly less invasive than traditional transcranial procedures with overall reduced morbidity, shortened procedure time, reduced hospital stay, and better cosmetic and lower recurrence rates. It should be considered as the preferred surgical alternative for initial treatment of symptomatic PACG but also for the treatment of symptomatic recurrence, independently of the initial surgical route. Repeat endoscopic endonasal surgery must be adapted to recurrent PACG. The infrapetrous approach with wider bony opening, extensive anterior cyst wall removal, and the use of a stent should be the treatment of choice for recurrent PACG.

\section{References}

1 Sincoff EH, Liu JK, Matsen L, et al. A novel treatment approach to cholesterol granulomas. Technical note. J Neurosurg 2007;107 (2):446-450

2 Eisenberg MB, Haddad G, Al-Mefty O. Petrous apex cholesterol granulomas: evolution and management. J Neurosurg 1997;86 (5):822-829

3 Griffith AJ, Terrell JE. Transsphenoid endoscopic management of petrous apex cholesterol granuloma. Otolaryngol Head Neck Surg 1996;114(1):91-94

4 Brackmann DE, Toh EH. Surgical management of petrous apex cholesterol granulomas. Otol Neurotol 2002;23(4):529-533 
5 Terao $\mathrm{T}$, Onoue $\mathrm{H}$, Hashimoto $\mathrm{T}$, Ishibashi $\mathrm{T}$, Kogure $\mathrm{T}$, Abe $\mathrm{T}$. Cholesterol granuloma in the petrous apex: case report and review. Acta Neurochir (Wien) 2001;143(9):947-952

6 Thedinger BA, Nadol JB Jr, Montgomery WW, Thedinger BS, Greenberg JJ. Radiographic diagnosis, surgical treatment, and long-term follow-up of cholesterol granulomas of the petrous apex. Laryngoscope 1989;99(9):896-907

7 Georgalas C, Kania R, Guichard JP, Sauvaget E, Tran Ba Huy P, Herman P. Endoscopic transsphenoidal surgery for cholesterol granulomas involving the petrous apex. Clin Otolaryngol 2008;33(1):38-42

8 Michaelson PG, Cable BB, Mair EA. Image-guided transphenoidal drainage of a cholesterol granuloma of the petrous apex in a child. Int J Pediatr Otorhinolaryngol 2001;57(2):165-169

9 DiNardo LJ, Pippin GW, Sismanis A. Image-guided endoscopic transsphenoidal drainage of select petrous apex cholesterol granulomas. Otol Neurotol 2003;24(6):939-941
10 Presutti L, Villari D, Marchioni D. Petrous apex cholesterol granuloma: transsphenoid endoscopic approach. J Laryngol Otol 2006;120(6):e20

11 Oyama K, Ikezono T, Tahara S, Shindo S, Kitamura T, Teramoto A Petrous apex cholesterol granuloma treated via the endoscopic transsphenoidal approach. Acta Neurochir (Wien) 2007;149 (3):299-302, discussion 302

12 Samadian M, Vazirnezami M, Moqaddasi H, Rakhshan M, Khormaee F, Ashraf H. Endoscopic transrostral-transsphenoidal approach to petrous apex cholesterol granuloma: case report. Turk Neurosurg 2009;19(1):106-111

13 Zanation AM, Snyderman CH, Carrau RL, Gardner PA, Prevedello DM, Kassam AB. Endoscopic endonasal surgery for petrous apex lesions. Laryngoscope 2009;119(1):19-25

14 Mattox DE. Endoscopy-assisted surgery of the petrous apex. Otolaryngol Head Neck Surg 2004;130(2):229-241 